\title{
Repensar la universidad: la didáctica, una opción para ir más allá de la inclusión de tecnologías digitales
}

\author{
Ángel Díaz-Barriga
}

\section{RESUMEN}

La pandemia sorprendió al conjunto de las sociedades, en un momento paralizó prácticamente las actividades que se realizaban de manera cotidiana. La educación, el trabajo docente en la universidad fue suspendido. La respuesta instrumental casi inmediata fue apoyarse en tecnologías digitales para continuar con las tareas de enseñanza, dejando aproximadamente al 25\% de los estudiantes de educación superior fuera. Sin embargo, la universidad no ha aprovechado este tiempo para estudiar los cambios que necesita realizar para vincularse con las demandas de la sociedad del siglo XXI. La pandemia mostró un aspecto de una crisis de la institución que ha perdido su horizonte, pues actualmente trabaja para mejorar sus indicadores en los programas de calidad, abandonando el análisis de su función civilizatoria. En su multicentenaria historia siempre ha realizado los cambios estructurales que cada época demanda, sin embargo en este momento sólo se plantea que basta con incorporar tecnologías digitales para transformarse, sin analizar las razones por las que las clases expositivas permanecen en sus aulas. Este ensayo plantea la necesidad de revisar la crisis fundamental que atraviesa, para reestructurarse de acuerdo con las condiciones del siglo XXI incluyendo la transformación de su docencia, apoyándose en seis principios didácticos, pues su futuro no puede sólo estar encadenado a una pantalla.

Palabras clave: crisis universitaria, reconstruir el sentido de universidad, re-inventar la docencia, tecnología digital, México. 


\title{
Repensar a universidade: a didática, uma opção para ir além da inclusão de tecnologias digitais
}

\section{RESUMO}

A pandemia surpreendeu ao conjunto das sociedades, em um momento paralisou praticamente as atividades que se realizavam de maneira cotidiana. A educação, o trabalho docente na universidade foi suspendido. A resposta instrumental quase imediata foi apoiar-se em tecnologias digitais para continuar com as tarefas de ensino, deixando aproximadamente 25\% dos estudantes de educação superior de fora. Contudo, a universidade não tem aproveitado este ano para estudar as mudanças que necessita realizar para vincular-se com as demandas da sociedade do século XXI. A pandemia mostrou a cara de uma crise da instituição que tem perdido seu horizonte, pois atualmente trabalha para melhorar seus prognósticos nos programas de qualidade, abandonando a análise de sua função civilizatória. Em sua centenária história sempre realizou as mudanças estruturais que cada época demanda, contrariamente neste momento só se projeta incorporar tecnologias digitais para transformar-se, sem analisar as razões pelas quais as aulas expositivas permanecem em suas salas. Este ensaio apresenta a necessidade de revisar a crise fundamental que atravessa, para reestruturar-se de acordo com as condições do século XXI incluindo a transformação de sua docência, baseando-se nos seis princípios didáticos, já que seu futuro não pode só estar acorrentado a uma tela.

Palavras chave: crise universitária, reconstruir o sentido de universidade, reinventar a docência, tecnologia digital, México.

\section{Rethinking the university: didactics, an option to go beyond the inclusion of digital technologies}

\begin{abstract}
The pandemic took society as a whole by surprise, practically paralyzing during a rather long while the activities that were carried out on a daily basis. Education and university teaching work was suspended. The almost immediate instrumental response was to rely on digital technologies to continue teaching, leaving approximately $25 \%$ of higher education students outside. Nevertheless, the university has not taken advantage of this year to study the changes it needs to make to link up with the demands of 21st century society. The pandemic showed one side of a crisis of the institution that has lost its way, as it is currently working to improve its indicators in quality programs but abandoning the analysis of its civilizing function. In its centenary history the University has always made the structural changes that each era demands; quite the opposite, at this moment it is only proposed that it is enough to incorporate digital technologies to transform itself, without analyzing the reasons why expository classes remain in its classrooms. This essay raises the need to review the fundamental crisis it is going through, in order to restructure itself according to the conditions of the 21st century. This includes the transformation of its teaching, based on six didactic principles, since its future cannot only be chained to a screen.
\end{abstract}

Key words: university crisis, reconstructing the meaning of university, re-inventing teaching, digital technology, Mexico. 


\section{Introducción}

Nunca en la historia de la humanidad una pandemia había afectado de una forma tan significativa a todos los sistemas educativos del orbe y al conjunto de instituciones universitarias, nunca antes se habían paralizado prácticamente todas las instituciones educativas; fue necesario buscar formas de trabajo escolar desde los hogares, aun cuando éstos no tuvieran las condiciones para realizar esta labor.

La necesaria respuesta instrumental de pasar prácticamente de la noche a la mañana a un trabajo docente mediado exclusivamente por tecnologías digitales, además de mostrar que la investigación en el uso de tecnologías en la educación ha dejado muchos temas pedagógicos pendientes, ha sido el espacio para evidenciar con mayor crudeza una crisis institucional de la universidad. Una crisis con respecto a sus finalidades, con respecto a la forma como responde a las condiciones que la sociedad le demanda, así como una crisis en el modelo didáctico que orienta su quehacer en las aulas.

La pandemia, que ha suspendido actividades docentes presenciales por más de un año, no ha sido aprovechada para examinar los problemas que emanan de la dinámica cotidiana de la institución, por el contrario, en una especie de obnubilación, se considera que abre las puertas para que en el futuro de la institución se mantenga la continuidad en su trabajo pedagógico ahora apoyado por el uso de tecnologías digitales. Por ello podemos afirmar que el trastocamiento ocasionado por la pandemia no ha sido empleado para analizar los problemas estructurales por los que atraviesa la institución universitaria. La tarea de analizar el futuro pedagógico de la universidad después de la pandemia no se puede realizar sin previamente hacer una reflexión sobre la necesidad de repensar la función que cumple esta institución en nuestra sociedad.

Este ensayo está compuesto de tres secciones, la primera examina algunos rasgos de la crisis que experimenta la universidad, en particular, la necesidad de analizar la forma como atiende a sus fines en el contexto del siglo XXI; la segunda propone la necesidad de re-inventar la forma como realiza su tarea docente, ante una serie de cambios sociales, cambios generacionales que experimentan los sujetos de la educación y ante el desarrollo de las tecnologías digitales, pero sin considerar que con éstas se resuelve el problema que se enfrenta en la actualidad. En el texto se cuestiona la falta de una perspectiva acorde al debate actual que se realiza en el campo de la didáctica, que transforme la manera de trabajar en el aula. Finalmente, la tercera parte propone seis principios didácticos que permitirían orientan esta re-fundación del trabajo docente en la institución universitaria.

\section{La universidad requiere re-pensarse a sí misma para enfrentar una crisis que precede a la pandemia}

La pandemia ha interrumpido de alguna forma la vida de las universidades, su vida presencial, con todas sus cualidades y limitaciones, con su dinámica habitual que invita tanto a la charla informal en los pasillos, como a los amplios debates académicos, a la realización de seminarios y trabajos docentes; ha afectado las áreas de investigación, los laboratorios y talleres, así como su dinámica cultural y deportiva. Si bien a través de las clases apoyadas en tecnología digital ${ }^{1}$ la institución tiene presencia, al mismo tiempo se puede reconocer su ausencia, un vacio cultural que en torno a ella se ha gestado. La riqueza de la

\footnotetext{
${ }^{1}$ En el texto prefiero emplear el término "tecnología digital" que la expresión "nuevas tecnologías", por dos razones: la primera es porque la tecnología se ha incorporado a la educación desde hace muchos siglos, el libro impreso es un resultado del descubrimiento de la imprenta en el siglo XV y segundo, porque las tecnologías digitales no son nuevas, en realidad hace más de 30 años que éstas se han venido conformado y su presencia en los sistemas educativos data ya de mucho tiempo, lo que no significa necesariamente que se efectúe un empleo didáctico de las mismas.
} 
cotidianidad de la vida universitaria ha sido cancelada de alguna forma, quedando restringida a una pantalla de computadora o de un smartphone. Como consecuencia, un buen número de estudiantes ha desertado debido a que no cuenta con ninguno de estos medios o con posibilidades de financiar la conectividad necesaria. El estudio del Instituto Internacional para la Educación en América Latina y el Caribe (IESALC, 2020) plantea que cerca del $25 \%$ de la matrícula en América Latina abandonará sus estudios por no contar con las condiciones para realizarlos en esta modalidad.

Las universidades, en la mayor parte de las regiones del globo y de América Latina, han dado una respuesta inmediata para intentar disminuir el riesgo de perder toda su docencia, rápidamente acudieron al empleo de diversas plataformas tecnológicas que permitieran continuar con la impartición de su compromiso docente. ${ }^{2}$ La respuesta instrumental fue de cierta forma necesaria pero no suficiente, porque no se ha aprovechado el impacto de la pandemia para estudiar los problemas fundamentales que la educación superior atraviesa en este momento; no se ha estudiado la forma en que su evolución y crecimiento, simultáneamente, han desvirtuado sus fines, ni tampoco se ha vislumbrado la necesidad de una reestructuración no sólo de las formas docentes, sino del conjunto de lo que se necesita conceptualizar como la institución universitaria del siglo XXI.

En realidad desde hace varios años la universidad está en crisis. Una crisis pedagógico-didáctica que no se desea reconocer. La docencia centrada en la explicación del profesor frente a un grupo de alumnos sigue siendo el modelo predominante del trabajo en el aula, los planes de estudio llenos de información de todo aquello que se supone que un profesional debe saber. Una visión enciclopédica ha permeado a estos planes y sólo el trabajo en la pandemia está obligando en algunas ocasiones a pensar cuáles son los temas sustantivos que un estudiante necesita conocer y, sobre todo, cuáles son los procesos intelectuales que requiere desarrollar para ser un profesional de cara al siglo XXI. Un profesional que enfrentará un mundo no sólo muy competitivo, sino con profundos y vertiginosos cambios en su trayectoria laboral. Aprender a aprender, aprender a resolver problemas, son temas postergados por políticas institucionales y nacionales, en las que el aprender se concentra en la capacidad de resolver con eficacia un examen a gran escala al egreso profesional. La universidad se ha convertido en una institución que se está negando a evolucionar y aunque algunos piensan que la gran transformación que tendrá en la pospandemia es la inclusión de la tecnología digital en el trabajo docente, necesita replantearse de fondo. En estricto sentido enfrenta una disyuntiva entre sólo modernizarse accediendo al mundo digital o transformarse atendiendo a lo que la sociedad espera de ella.

Pero para pensar en el futuro de esta institución, cuyos inicios se remontan al siglo XI, es indispensable iniciar desde un análisis que vaya más allá de lo pedagógico, dónde se evalúe hasta dónde en este momento responde a las condiciones de las sociedades de este siglo. Tengamos en cuenta que esta centenaria institución ha sido capaz, a lo largo de su existencia, de realizar diversas transformaciones en función de lo que cada etapa histórica de la sociedad le ha requerido. En este sentido la situación actual reclama, como en otras ocasiones críticas, construir una propuesta de cambio mucho más profunda en la institución.

Freitag (2004) sostiene que la universidad a lo largo de su historia ha sabido adaptarse a diversas condiciones sociales. Podríamos incluso tomar como referencia

\footnotetext{
${ }^{2}$ Intencionalmente utilizo la expresión "impartición de su docencia” y no trabajo docente porque desde un punto de vista didáctico hay una diferencia sustantiva en ello. Muchos elementos de una acción didáctica como son la interacción, el vínculo pedagógico o la construcción colectiva del conocimiento, sencillamente se dejaron de lado o se acentuó su ausencia en las prácticas de los docentes de educación superior, muchos de los cuales sólo asumen que su trabajo consiste en exponer conocimientos o dictar cursos.
} 
lo realizado en los años posteriores a la ruptura social del movimiento estudiantil de 1968, que afectó a México y a otros países. Una ruptura con una forma de gobierno, aunada a una demanda de democratización que no sólo tuvo impacto en la vida pública del país, sino que posibilitó una reestructuración en algunos sentidos de la universidad. En este contexto sobresalieron dos formas de concretar el cambio, en primer término reconocemos a un conjunto de instituciones que buscaron articular un cambio en la estructura institucional con un significativo impacto pedagógico, mientras que otras instituciones sólo se quedaron en la conformación de un proyecto político que no logró bajar a un cambio en la forma didáctica como se realiza el trabajo en el aula.

En el primer caso se encuentran proyectos institucionales que buscaron que la formación profesional realizada en sus aulas se vinculara con la resolución de algunos problemas de los sectores de mayor carencia en la sociedad. Estos proyectos lograron modificar sus planes de estudio, concretando en algunos casos proyectos de integración curricular (Díaz Barriga, 2020) como el sistema modular de la Universidad Autónoma Metropolitana (UAM)-Xochimilco, el del Centro Interdisciplinario de Ciencias de la Salud, del Instituto Polítécnico Nacional, el proyecto de Medicina Integral A-36 de la Universidad Nacional Autónoma de México (UNAM) o el plan de estudios de la Facultad de Arquitectura, organizado por talleres, así como la creación del Colegio de Ciencias y Humanidades (CCH), ambos de la UNAM. Estos planes de estudio lograron articular tres aspectos: superar la división tradicional de disciplinas y trabajar por problemas; transformar el trabajo en el aula descentrando la función docente de sólo transmitir información; y vincular el aprendizaje de los estudiantes a la atención de algunos problemas de los sectores mayoritarios de la sociedad.
Una nota particular que en este momento también sería relevante tener en cuenta es la creación del Sistema Universidad Abierta (SUA) en la UNAM, sistema que en su momento fue ideado para ofrecer un servicio educativo a aquellos trabajadores que no habían logrado realizar sus estudios universitarios. El SUA no necesariamente fue concebido como una universidad paralela, sino como una forma de articular a profesores, estudiantes y trabajadores. Tema que, en opinión del ex rector que promovió la fundación del sistema, no se ha logrado (González Casanova, 1976).

El segundo grupo de instituciones universitarias creadas en ese momento buscó abandonar el modelo de facultades y escuelas y transitar hacia una estructura departamental, como el caso de las unidades que conforman la Universidad Autónoma Metropolitana (UAM) o el modelo desarrollado por la Universidad Autónoma de Aguascalientes. Mientras que en otras prevaleció básicamente un proyecto político como el proceso que siguieron varias universidades denominadas democráticas, críticas y populares, caracterizadas por la búsqueda de una estructura democrática, en general dominadas por grupos vinculados al Partido Comunista - no reconocido oficialmente en el país - y que incluso llegaron a ser controladas por movimientos extremos como el denominado "Los Enfermos" en la Universidad Autónoma de Sinaloa (Sánchez, 2007). Es necesario reconocer que estas instituciones no lograron transformar la práctica docente centrada en la exposición, sino que sólo incorporaron en sus planes de estudio alguna materia de corte marxista.

Necesitamos reconocer que la ruptura del orden social generada por el movimiento del 68 fue el escenario para impulsar un cambio en varios casos estructural, tanto en la organización de la universidad como en la elaboración de sus planes de estudio, así como en el trabajo que se realiza en las aulas. ${ }^{3}$

\footnotetext{
${ }^{3}$ No sólo en México se experimentaron este tipo de cambios. La Universidad de París fue rearticulada en varios campus dando origen a otras instituciones París I, II, III, IV, etcétera.
} 
Hoy estamos ante un evento que incluso tiene una magnitud mayor al anteriormente señalado, pues la afectación social, educativa y de salud que ha generado la pandemia tiene efectos globales. No sólo se trata de ver el caso de la sociedad mexicana, sino de reconocer que este impacto global es un espacio para volver a analizar hasta dónde la institución universitaria necesita salirse de las políticas hacia la educación que actualmente la tienen ahogada, como las que han emanado de las llamadas políticas de calidad para la educación superior, que han sido impulsadas en los últimos 40 años tanto por organismos internacionales (Banco Mundial y la OCDE) como por gobiernos locales. Analizar hasta dónde la búsqueda de indicadores de calidad le ha impedido visualizar cuál es el proyecto con el que busca vincularse con la realidad social.

Por ello, la discusión universitaria no puede reducirse sólo al estudio de tecnologías digitales o cómo será el trabajo mixto una vez que la pandemia haya sido controlada dentro de algunos años. La universidad no puede desperdiciar el momento de re-pensarse a sí misma, de re-valorar cuál es su función social.

En su libro el Naufragio de la universidad, Freitag (2004) previene sobre la necesidad que tiene la institución universitaria de realizar los cambios que cada época le demanda, con el fin de cumplir con su responsabilidad y trabajar por su sobrevivencia. Ése es quizá uno de los grandes temas pendientes de nuestra institución actual. La sociedad mexicana atraviesa desde hace muchos años por diversas crisis, 40 años atrás vivió una económica con su significativo impacto social, creando amplios grupos de pobreza, los que lamentablemente se han mantenido y acentuado hasta hoy. Tenemos un país que, como resultado de la pobreza, ha visto incrementada la violencia de una manera exponencialmente creciente, en diversos grados que muestran claramente degradación social. Éstos y otros problemas de su entorno, no forman parte del debate universitario actual, como si la calidad académica fuese opuesta a cumplir con la indispensable función civilizatoria.

Una universidad que se sostiene de los recursos públicos, que realiza una importante tarea de formación de profesionales de muy diversas especialidades, que realiza tanto investigación básica como aplicada, así como indagación en un importante número de áreas de conocimiento, que tiene proyectos de difusión cultural, hoy necesita pensar si su organización y estructura responden a las exigencias de la sociedad actual; necesita revisar cómo reestructurar su organización para atender de mejor manera sus finalidades, de suerte que no sólo sea una institución del siglo XXI, sino se convierta en una institución que se transforma para responder al momento crucial que vivimos.

La universidad mexicana requiere revisar a fondo la forma como ha sido afectada en los últimos 40 años por un conjunto de programas concebidos bajo la égida de la calidad, en donde actores externos a la universidad - como el sistema nacional de investigadores, los diversos organismos acreditadores de planes de estudios, el establecimiento de programas de estímulos al desempeño académico, el papel que juegan los sistemas de exámenes de ingreso y egreso - han afectado su dinámica interna de trabajo.

Hoy conseguir puntos y calificaciones se ha convertido en la meta del trabajo académico de la institución y de su planta académica. El horizonte de la vida universitaria ha quedado atrapado en la obtención de diversos puntajes para afirmar que ha dado respuesta a las políticas de calidad, nacional e internacional; esta búsqueda por elevar las métricas ha deformado y limitado su función. Esta institución define sus proyectos de formación profesional buscando la atención y cualificación en estos programas, en los diferentes rankings que se hacen para comparar a las instituciones universitarias a nivel global, en detrimento de analizar cuál es su proyecto académico, su sentido social, su función civilizatoria en la sociedad en que se encuentra inmersa. 
Parecería muy corto de miras pensar que sólo con la incorporación de la tecnología digital se adecuará al replanteamiento institucional requerido. Si la universidad considera que su futuro se encuentra únicamente en tecnologizarse, en implantar un modelo de educación semipresencial, en donde parte de su tarea se realice con el uso de diversas aplicaciones, es una institución que se está negando a pensarse críticamente a sí misma. Si considera que el reto mayor que enfrenta es cómo "capacitar" a su personal académico en el uso de diversas plataformas digitales da un ejemplo deprimente de cómo ha abandonado su función social y académica.

El futuro institucional y pedagógico de la universidad se encuentra comprometido, pero la pandemia podría y tendría que ser el espacio para convocar a los universitarios a una cantidad exponencialmente creciente de mesas redondas, en las que se analice cuáles son los cambios estructurales que requiere la institución y cómo repensar sus finalidades. En estos meses hemos mostrado amplia incapacidad para convocar a seminarios, para analizar la crisis sistémica por la que atraviesan nuestras instituciones, la crisis interna; estamos mostrando dificultad para repensar los fines de la institución; proponer una reestructuración que nos permita cumplir con las funciones que las condiciones del siglo XXI y la pandemia demandan, en el marco de la diversidad de nuestras sociedades.

La respuesta que la universidad ha tenido en el contexto de la pandemia muestra que ha logrado una cierta eficacia en mantener de alguna forma, no necesariamente plena, pertinente y didáctica, su actividad docente y de investigación. Pero al mismo tiempo es un ejemplo de una incompetencia para re-pensarse, para buscar otras formas que le permitan cumplir su compromiso con la sociedad que la sostiene. Lo importante, lo relevante ante la suspensión de actividades presenciales ha sido encontrar una forma de continuar con los planes y programas de estudio como si no hubiese acontecido nada. $\mathrm{Ni}$ siquiera ha mostrado capacidad de emplear a plenitud la potencialidad que ofrece la tecnología digital, ni necesariamente atender a los requerimientos de los estudiantes, tanto en las actividades de trabajo académico para su formación, como en las posibilidades de contectividad indispensables para realizar su labor. Incluso proponer seis o más horas frente a una pantalla, más la necesidad de cubrir una serie de lecturas y tareas, no es responder a un proyecto de trabajo didáctico acorde con las condiciones de nuestro siglo. Pensar que el futuro de la institución se encuentra en la pantalla realmente es un ejemplo de la cortedad de miras en las que vive la universidad en este momento.

\section{Romper con la continuidad pedagógica en la pandemia y después de ella. Las tecnologías digitales no necesariamente lo logran}

La actividad docente seguramente es la que ocupa la mayor parte del esfuerzo académico que realiza la universidad, la mayor parte de su planta académica y obviamente miles de estudiantes que la integran tienen como actividad central la tarea educativa que se realiza en los salones de clases, laboratorios y talleres. Aunque la vida universitaria no se puede reducir a ello.

Cuanta razón tiene Agamben (2020) al sostener, en un breve ensayo titulado Requiem por los estudiantes, que frente a la pantalla los estudiantes universitarios han muerto, dejaron de vivir lo que significaba estar en la universidad. El trabajo que se realiza por medios digitales no puede suplir de ninguna manera la intensidad de la vida universitaria. La pantalla calla las discusiones cotidianas que se dan en el seno de la universidad, tanto las que surgen de manera espontánea en los pasillos, como las intensas que pueden darse en los salones de clase o en los diversos actos académicos; todo ello sucumbe ante una "barbarie tecnológica" donde la experiencia de los sentidos queda aprisionada por una "pantalla espectral", en 
expresión del filósofo. Los rectángulos de Zoom son el mejor ejemplo de cómo queda atrapado cada uno de los actores docentes y alumnos en un lugar inmóvil donde es difícil relacionarse, donde la comunicación viva y cotidiana desapareció.

Para Agamben (2020) la pérdida mayor es para los estudiantes, los que denomina el scholarii, quienes en muy diversos lugares tienen un espacio de encuentro en el escenario real de la institución. Este encuentro intenso, en opinión del autor, acontece desde los estudiantes universitarios medievales hasta los movimientos estudiantiles del siglo $\mathrm{XX}$, cada uno de nosotros puede dar un testimonio personal al respecto. Pero el autor plantea un asunto todavía más de fondo: las universidades se ahogaron en sí mismas, no fueron capaces de adaptarse a las diversas exigencias de su tiempo, y actualmente la tecnología logra modelar su fin y, podríamos añadir, se ahogaron en los puntajes que conceden los diversos programas de calidad. La institución que hoy se vislumbra en lo que será la pos-pandemia quedará cercenada de forma mediana o ampliamente por la tecnología digital. Vive una contradicción enorme, se moderniza aparentemente para perderse a sí misma.

Más aún, se considera que la universidad de la pospandemia recuperará su docencia presencial tal como la conoce, ahora compartida con la que se realiza en línea. Resulta por lo menos preocupante que en lugar de analizar la necesidad, entre otras tareas, de re-inventar el trabajo docente (Díaz-Barriga, en prensa), se considere que la renovación de la actividad docente será unicamente el resultado de incorporar en su actuación tecnologías digitales, para utilizar diversas aplicaciones como las que provienen de Google education, así como en la habilidad para subir a Classroom las tareas, las lecturas que hay que realizar durante el curso. Esta última plataforma permite rigidizar la tarea pedagógica al funcionar a través de un control estricto de la fecha y horario para que los alumnos suban sus trabajos o tareas, permitiendo al mismo tiempo al docente administrar exámenes elaborados por él o seleccionados de la plataforma, y colocar las calificaciones de los alumnos. Cierto, se logra orden de la actividad docente, pero al mismo tiempo tiempo se pierde el sentido didáctico de esta tarea. Apoyarse en tecnologías para que el docente siga siendo el centro del trabajo educativo, donde se pida a los alumnos que respondan o hagan determinadas tareas no es transformar la docencia. La plataforma Moodle permite, entre otras cosas, monitorear (controlar) los tiempos en los que los alumnos están conectados y las actividades que realizan; en el fondo la tecnología se convierte en el mejor sistema de vigilancia que haya sido ideado, un panóptico foucaltiano que ya ha sido enunciado para la educación (Foucault, 2009). La tecnología al servicio del control del trabajo educativo, en detrimento del estudio de los procesos que es necesario realizar para aprender.

La actividad docente es resuelta por una tecnología que lejos de permitir replantear lo que significa la tarea de generar condiciones de aprendizaje, rigidiza el trabajo y la relación de docentes y estudiantes. No se interroga sobre el papel del éxito en el proceso de formación o en el trabajo educativo (Freinet, 1993; Meirieu, 2006), sino que en su lugar se coloca la eficacia desprendida del viejo modelo de control de tiempos y movimientos tayleriano, hoy abruptamente tecnologizado; una plataforma que responde a un modelo tecnocrático para fortalecer el modelo de "soy un docente exigente", que con escándalo hemos visto presente en las redes sociales en las que los estudiantes denuncian situaciones negativas que viven actualmente con algunos maestros en el uso de la tecnología digital.

Cierto, durante la pandemia hemos sido testigos del gran interés que tuvieron las autoridades universitarias por reestablecer las clases, no la vida universitaria. La tecnología permitió dar una respuesta instrumental necesaria ante la crisis, pero insuficiente en dos dimensiones: ha servido para clausurar o al menos ocultar la necesidad de estudiar 
cuáles son los problemas estructurales que enfrenta nuestra institución, y al mismo tiempo, ha eliminado la posibilidad de analizar los cambios necesarios que hay que realizar en el trabajo docente desde una perspectiva didáctica. A esto hay que añadir que se ha acentuado la desigualdad social, una de cuyas expresiones es hoy la brecha tecnológica, ante la carencia de equipos o de conectividad, tema que trabajaremos un poco más adelante.

Se ha optado por "capacitar" a los docentes en el uso de diversas plataformas digitales, tanto las que se encuentran en acceso abierto como Meet o Hangouts, hasta las que se pueden ofrecer mediante un contrato como Zoom, Classroom, Blackboard, entre otras. El uso de tecnologías digitales no se realiza atendiendo a una perspectiva didáctica, en general se están empleando para reproducir el modelo de docencia centrado en la exposición, en la realización de tareas o trabajos y en la formulación de preguntas a los alumnos. Una tecnología de frontera en el siglo XXI para apoyarse en una didáctica propia del siglo XVII.

No se trata de descalificar la respuesta instrumental de impulsar un trabajo educativo a través de la pantalla, sino de reconocer que esta respuesta es insuficiente frente a la necesidad de analizar los problemas estructurales que emanan de una ausencia de análisis sobre la forma como la universidad en este momento responde a su finalidad, para de ahí derivar los cambios sustantivos que requiere institucionalmente, en su organización, así como en su trabajo docente desde una visión pedagógica (Tenti 2020). Ciertamente necesitamos reconocer y aceptar que la tecnología digital acompañará a la educación en el siglo XXI, pero acompañar no es sinónimo de que ella por sí misma transformará ni la educación, ni el acto didáctico.

No es la primera ocasión en que la tecnología se incorpora al acto educativo, a principios del siglo XX lo fue la radio educativa, posteriormente la televisión, con el desarrollo de la psicología conductual se desarrollaron las máquinas de enseñar en los años treinta del siglo pasado y posteriormente la enseñanza programada. En el furor de este movimiento Keller (1968) se apresuró a publicar su ensayo "Good bye Teacher...", el desarrollo tecnológico obtenido hasta ese momento pronosticaba que en el acto educativo se podría prescindir del docente. Todo estaba resuelto por la graduación del contenido que podía ofrecer una máquina de reforzamiento o la traducción del mismo a un texto construido bajo esa lógica.

Lejos estaba la década de los sesenta del siglo pasado de poseer el poder y capacidad que la tecnología digital ha desarrollado recientemente. Sin embargo, en el imaginario social, se empezaba a configurar la imagen de prescindir del docente. Imagen que con el tiempo se fue diluyendo, aunque en algunos momentos se ha presentado de otra forma: ofrecer materiales escritos o en línea de tal forma que "aún con malos docentes" los alumnos aprendan (Banco Mundial, 1990), hasta la idea de reencauzar el trabajo que realizan los docentes a través de una pantalla. En la era digital se llega a considerar que el trabajo escolar presencial o a distancia, apoyado en lo que se define como la nueva ecología del aprendizaje (Coll, 2013), permitirá que la educación finalmente llegue al culmen de su modernidad. Las personas quedan subsumidas y la didáctica sencillamente deja de existir.

\section{Avanzar hacia una visión didáctica en el uso de las tecnologías}

Insistimos en este ensayo que la institución universitaria necesita renovarse, pero ello incluye también analizar hasta donde la docencia también requiere transitar hacia otras formas de ser concebida y desarrollada. La didáctica es una disciplina para el docente, pero es una disciplina que desde su origen

\footnotetext{
${ }_{4}^{4}$ Por demás resulta interesante que se emplee el verbo capacitar a los docentes, verbo que desprofesionaliza la profesión, se capacita al tornero, al técnico medio. A los profesionistas se les ofrece educación continua o programas de actualización.
} 
en el siglo XVII se ha venido enriqueciendo y transformando, buscando adecuarse a las necesidades de cada etapa de la sociedad. Tanto Durkheim (1992) como Dilthey (1965) sostienen que un trabajo didáctico sólo se puede entender o juzgar en el contexto del momento social en donde éste acontece, plantean que en cada momento histórico se han conformado propuestas de enseñanza específicas que fuera de su tiempo se encuentran desfasadas. Este tema didáctico con mucho mayor detalle lo desarrolla Snyders en su texto Pedagogía progresista (1972).

Estableceremos seis principios didácticos que es importante tener en cuenta para este proceso de renovación de la docencia en el futuro de la educación superior, sin importar que la misma se realice en forma presencial o a distancia, pero partiendo de la idea de que es la reinvención del trabajo docente la tarea fundamental del futuro de la universidad y no solamente el uso de las tecnologías digitales en la pospandemia.

\section{Primer principio. La didáctica es una disciplina, la enseñanza es una tarea especializada}

El trabajo docente necesita adecuarse a cada momento social. La didáctica como disciplina ha tenido una evolución importante. Los planteamientos que reflejan el debate didáctico contemporáneo tienen múltiples rasgos referidos tanto a los docentes, como a los alumnos. Sin embargo, quizá es en el campo de la didáctica donde permea con más frecuencia un pensamiento claramente derivado del sentido común.

Este tema tiene su origen en dos elementos: por una parte en la falta de reconocimiento de que la tarea de enseñar es una actividad profesional especializada, que reclama formación específica y, en segundo término, en una falta de rigor conceptual en el campo de la didáctica que impide identificar las diferentes escuelas de pensamiento que la conforman.
La simplificación del pensamiento didáctico, que acompaña al mismo término de enseñanza, tiene como consecuencia no sólo que se acepte que cualquier persona puede enseñar y que basta obtener cualquier formación en un campo de la educación superior, para poder realizar la tarea de enseñar. Se expresa también en una aceptación de colocar el enunciado "didáctica" a múltiples expresiones socialmente aceptadas, entre otras "es una forma didáctica", "es un trabajo didáctico", "es una ejemplificación didáctica", expresiones que implican una presentación con falta o ausencia de rigor conceptual, pero que en la divulgación de un tema se supone que ayudan a que el mismo "sea comprendido por distintas personas".

En este sentido hacer algo didáctico no sólo es simplificarlo, sino deformarlo de tal forma que para su conocimiento no haya necesidad de contar con antecedentes conceptuales. Por ello, la palabra didáctica se suele emplear en varias ocasiones como un complemento de algo que no requiere una formación en una disciplina educativa específica.

A ello se auna la segunda cuestión, la conformación de la disciplina didáctica no ha logrado enunciarse a sí misma en una forma tan consistente que permita identificar tanto la construcción epistémica y conceptual que subyace en la misma, como las diversas escuelas o corrientes de pensamiento que a lo largo de los siglos la han conformado, tal como lo plantea Snyders (1972). Mucho menos atender con rigor a la compleja evolución que ha tenido en los últimos 50 años, en los que se han desarrollado con mayor claridad diversas escuelas de pensamiento, no necesariamente complementarias.

Este último aspecto se observa en los cursos que se trabajan relacionados con esta disciplina, cuyos contenidos se encuentran atrapados únicamente en temas vinculados con la metodología de enseñanza, en satisfacer la pregunta, ciertamente válida, sobre cuál es la mejor manera de enseñar determinado tema. 
La didáctica, al permanecer atrapada en una expectativa metodológica, se constituye en un obstáculo epistemológico (Bachelard, 2004) que impide visualizar el sentido intrínseco del debate sobre procesos de formación, así como las necesidades y condiciones de aprendizaje del ser humano. Es interesante reconocer que mientras en otras disciplinas, como psicología, sociología o filosofía, se reconoce la existencia de diversas escuelas de pensamiento, ${ }^{5}$ en el caso de los saberes didácticos éstos se enseñan de manera plana, como si no existiera un debate intenso entre las diferentes escuelas, de corte francófono, latinoamericano, alemán, italiano, etcétera. No se reconocen las diversas escuelas de pensamiento didáctico, que reflejan distintas posiciones y perspectivas de pensamiento, que dan origen a construcciones conceptuales específicas, diferentes y que no necesariamente convergen unas con otras. Hace medio siglo Snyders (1972) planteaba que el futuro del pensamiento didáctico estaría en avanzar en una perspectiva de síntesis entre las escuelas que en ese momento reconocían. Seguramente la construcción conceptual de las escuelas didácticas actuales no necesariamente responde a esa idea de síntesis, sino que se ha avanzado por rutas conceptuales específicas para analizar los procesos que subyacen en el trabajo docente, así como en las necesidades y procesos de aprendizaje de los alumnos.

Reconocer este tema es fundamental para la universidad con el fin de analizar lo que significa renovarse en su trabajo docente o quizá mejor expresado, re-inventar la docencia ante las exigencias del siglo XXI (Diaz-Barriga, en prensa).

\section{Segundo principio. La docencia es una actividad compleja}

Es necesario reconocer la complejidad del trabajo docente, de la diversidad de sus saberes, en particular, reconocer que su experiencia presente y pasada es definitoria de su práctica; ésta se entremezcla con la formación conceptual en el ámbito de la pedagogía, del currículo y de la disciplina que trabaja (Tardif, 2010). Tardif considera que la práctica docente es una actividad compleja, no sólo por las diferencias que existen en cada contexto escolar, sino por las diversas condiciones en las que se envuelve el mismo desempeño docente, en el que es necesario preguntarse qué necesita el alumno para realizar un aprendizaje, pero también responder a los saberes que emanan de una disciplina (el tema de su enseñanza), a un proyecto curricular específico y a un conjunto de condiciones institucionales donde se desenvuelve su labor.

La complejidad que emana de este conjunto de elementos constituye un reto para la realización de su tarea. Según este autor el docente, en la realización de su trabajo ya frente a sus estudiantes, no busca coherencia teórica, sino encontrar una respuesta práctica a la situación que enfrenta (Tardif, 2010). Esto no significa que un docente se pueda seguir refugiando en el modelo de trabajo expositivo que caracteriza su labor, ni necesariamente constituye una negación de la necesidad de realizar un acto de transmisión de información, como parte de su tarea. En este momento se experimenta el reto de encontrar la forma de dosificar la transmisión con el establecimiento de una serie de espacios diversos para que los estudiantes puedan desarrollar su proceso de aprendizaje.

Sostener que la práctica docente no responde a una coherencia teórica sino a una exigencia práctica, no implica que debamos mantener el modelo expositivo de enseñanza por ser el único que se domina, ni tampoco asumir la necesidad de trabajar bajo modelos que prescriben lo que hay que realizar en la tarea docente, pues la lucha de la didáctica por salirse de

\footnotetext{
${ }^{5}$ Conductismo, constructivismo, Gestalt en Psicología; empirismo, funcionalismo, estructuralismo, marxismo en Sociología; filosofía griega, tomista, cartesiana, kantiana, existencialista en Filosofía.
} 
las prescripciones ha sido una batalla de largo tiempo. Tampoco quiere decir que entonces no existen principios didácticos que el docente necesita conocer para tomar las decisiones profesionales que requiere para enfrentar su trabajo para con sus estudiantes, buscando generar condiciones de aprendizaje. Precisamente en relación con todos estos elementos se puede entender que el trabajo docente demanda respuestas concretas a situaciones específicas que no pueden ser prescritas ni pensadas en su totalidad de antemano.

En este sentido, parte de la complejidad de las decisiones que tiene que tomar el docente surgen de analizar qué requiere el estudiante para aprender, analizar cuáles son las condiciones y posibilidades de cada grupo de estudiantes y con estos elementos idear una forma de trabajo. Cada grupo tiene características singulares, la secuencia didáctica seguida durante una clase no puede ser la misma para todos los grupos. El docente tiene que examinar cómo evoluciona el grupo, promover la reflexión de los alumnos sobre su trabajo, sobre sus avances, dificultades e incluso su falta de compromiso. La docencia reclama de experimentación permanente si se desea realizar como una actividad rigurosa. La reflexión y análisis sobre la situación generada en el trabajo del grupo es un elemento sustancial para poder generar condiciones de aprendizaje. En este camino se desconoce la ilusión que socialmente se asigna a la docencia de concebir que hay una "clase modelo" que todos los que se dedican a la enseñanza deben seguir.

Así, la idea de la existencia de un método de enseñanza se quiebra y al mismo tiempo se transforma, ya que deja de considerarse como una serie de pasos rígidos que hay que cumplir, por el contrario, la estrategia metodológica se concibe como el resultado de la integración de diversos principios que permanentemente se adecuan en el aquí y ahora de cada grupo escolar. No hay un método, sino una estructura de pensamiento didáctico que ofrece principios explicativos y orientadores para el trabajo docente.

Pero corresponde a cada docente no sólo elaborar una planeación para el trabajo a realizar con cada grupo escolar, al mismo tiempo necesita aceptar que cuando se encuentre en la situación didáctica, en el aquí y ahora con sus estudiantes, esa planeación se reestructurará de acuerdo con las circunstancias que en ese momento considere como la mejor opción para crear las condiciones de aprendizaje. Siempre como una hipótesis que orienta su trabajo.

\section{Tercer principio. Trabajar un contenido a través de un problema}

Particular atención es necesario conceder a vincular los contenidos con problemas cercanos a la vida real, de suerte que los alumnos se los puedan apropiar como algo que le conceda significatividad al tema que se va a trabajar. Entender que no se trata de crear motivaciones, muchas de ellas referidas a aspectos superficiales de la vida del sujeto, sino de intentar conectar el interés del alumno con un problema, de tal forma que éste pueda constuir el aprendizaje como un reto, como un proyecto personal (Meirieu, 2002). Así, desde su fundación la didáctica reconoció que la voluntad del alumno no puede ser obligada (Comenio, 1982), esto es, que no se puede provocar el aprendizaje ante un alumno que sencillamente no quiere o no desea aprender (Freinet, 2009). ${ }^{6}$ Aprender siempre demandará un esfuerzo, pero este esfuerzo sólo puede efectuarse a partir de que el estudiante asuma que desea aprender. Cualquier estímulo que sólo permanezca en lo externo del individuo, en lo que generalmente se denomina motivación, sólo provocará una atención secundaria en la tarea que se demanda.

Pero no hay que confundir crear un ambiente de aprendizaje, con simplificar los contenidos a trabajar

\footnotetext{
${ }^{6}$ Freinet (2009) escribió el ensayo "Dar de beber a un caballo que no tiene sed" para señalar que los alumnos sólo aprenderán algo cuando consideren que necesitan ese saber.
} 
en un curso. No es facilitar la tarea en el sentido de solamente hacerla sencilla, sino en facilitar la posibilidad de que el alumno arme su proyecto de aprender, que reconozca su voluntad y decisión frente a esta tarea. Ciertamente, como lo expresa Chevallard (1997), es pasar del saber sabio al saber enseñado. De alguna manera, esto implica que el docente tenga claridad sobre el esfuerzo que demanda de sus alumnos, sobre el reconocimiento de que el alumno sólo aprende a partir de lo que realiza (Aebli, 1958) y, al mismo tiempo, analizar en qué momentos el docente decide cumplir con la tarea de transmisión, en el papel de quien contiene el saber, en el sentido de Tardif (2010), disciplinario, curricular, pedagógico y experiencial. De ahí la necesidad de considerar la dimensión experimental del trabajo docente.

\section{Cuarto Principio. Las múltiples interacciones en el proceso de aprender}

Un principio didáctico que necesariamente acompaña a la tarea de construir situaciones de aprendizaje se encuentra vinculado con la necesidad de la interacción. La interacción, en primer lugar entre cada estudiante con un tema u objeto de aprendizaje, pero en la tarea de enseñar esa interacción también se debe realizar entre el docente y los estudiantes; entre los estudiantes en pequeños grupos, o al azar con alguno de sus compañeros. La interacción es fundamental ya que permite generar un necesario diálogo en el acto de aprender, diálogo que permite realizar interrogaciones, aclaraciones, visualizar el objeto de aprendizaje desde diversas ópticas. Diálogo es quizá el tema más antiguo del trabajo de aprendizaje, que antecedió por siglos a la construcción del pensamiento didáctico; en el fondo, la mayeútica socrática de eso se trataba.
Incluso podemos reconocer que cuando los estudiantes, e incluso cualquier persona, dedica mucho tiempo en una computadora a una búsqueda, por ejemplo en Google, de algún tema que por alguna razón le interesa, ese tiempo se alarga precisamente por las interacciones que en esa búsqueda realiza. La interacción es un tema clave, pero no necesariamente se puede impulsar de manera mecánica. Requiere de la voluntad del estudiante, pero también de la capacidad del docente, en forma presencial o por medios digitales, para promover un intercambio de ideas, un diálogo, que si bien en general emana de algo que el docente hace o pide hacer, sólo puede ser resultado de un compromiso de los estudiantes con alguna tarea específica.

Por ello afirmamos que la didáctica no puede modelar la forma de trabajar, ni de los docentes, ni de los alumnos. Éste es un reto que no se puede desconocer en las clases que se realizan sólo con soporte digital en la pandemia e incluso en la educación a distancia. De ahí que pensemos que las aulas (cuando regresemos a ellas) tendrían que tener una disposición para la interacción en múltiples sentidos, quizá pensar incluso en una estructura física que ya no sea rectangular, que ya no tenga la disposición de un docente frente a unos alumnos sentados en serie, sino que su estructura y mobiliario permita al docente transitar por diversos espacios del salón de clase, y a los estudiantes contar con una organización que permita el trabajo individual, en pequeños grupos o en el grupo completo. ${ }^{7}$

\section{Quinto principio. Construir un vínculo pedagógico}

Junto con este tema es conveniente también resaltar la necesidad de construir un vínculo pedagógico. La

\footnotetext{
${ }^{7}$ Observar la concepción, propuesta pedagógica y construcción de las aulas en modelo octagonal del Centro Universitario Valles de la Universidad de Guadalajara, permitiría analizar la necesidad de cómo, al replantear el trabajo docente, también se modifica sustantivamente la estructura física de la construcción del aula escolar; esta experiencia se puede observar en YouTube, "Recorrido instalaciones del CUValles, 2010 (https://www.youtube.com/watch?v=qtgrSeFgtlw).
} 
noción de vínculo es mucho más fuerte y reclama un compromiso de mayor identificación entre los sujetos vinculantes, que la mera relación docente-alumno. Cierto que el vínculo parte de reconocer la existencia de esa relación, pero va más allá, pues no sólo se trata de la identificación mutua que acontece en la relación mediada por la enseñanza, entre un conjunto de sujetos que se reconocen como parte de la misma y se identifican en una dinámica, siguiendo libremente el pensamiento de Pichón-Rivière (1980); en el vínculo se produce una dinámica entre elementos internos y externos de los sujetos involucrados.

Este acto de mediación se constituye a partir de un complejo interjuego entre un docente que se encuentra en condición de dar u ofrecer información y, una serie de sujetos que aceptan el reto de trabajar con esa información para generar un proceso de construcción activa de la misma. En esa compleja dinámica se construye un vínculo pedagógico relacionado genéricamente con las condiciones, los objetos y los múltiples aprendizajes. Un encuentro dinámico en el que paradójicamente, a partir de la propuesta de un tema para ser enseñado o transmitido, se generan intercambios entre todos los actores, esto es, entre docentes y alumnos que relacionan muy diversos saberes previos de ambos, que posibilitan la construcción de un aprendizaje, no del aprendizaje, pues el aprendizaje construido por cada uno de los actores involucrados necesariamente responde a sus historias y procesos personales. Así, en el proceso intencional que se desprende de una relación entre un docente y un conjunto de estudiantes que de forma individual y colectiva se van relacionando con el objeto de esta información, se generan procesos de aprendizaje diferenciados en todos ellos. Estos procesos son resultado de la posibilidad de construir un vínculo pedagógico.
Este vínculo se desprende de la interacción que se realiza entre docentes y alumnos, lo que implica un reconocimiento mutuo de quiénes se encuentran en una situación de aprendizaje intencionado. Si bien no surge de manera inmediata del reconocimiento del trabajo docente, sí corresponde al docente crear condiciones para que este vínculo pedagógico se conforme a través de promover un clima de reconocimiento, como sujetos que participan en una situación de aprendizaje escolar. Esto se deriva de alguna forma de la manera como el docente se dirige hacia los estudiantes, de sus saberes profesionales y pedagógicos, del reconocimiento de la existencia de saberes de sus estudiantes, de asumir que toda situación de aprendizaje abre una posibilidad de aprender para el estudiante, pero no necesariamente obliga su voluntad.

El vínculo pedagógico es el reconocimiento de una relación mutua, en donde de diversa manera docentes y alumnos enfrentan los retos y esfuerzos para desarrollar un aprendizaje. Este vínculo no surge de manera mecánica, pero es uno de los elementos de mayor especificidad en la relación didáctica. Sin embargo, el aula virtual, el trabajo a distancia constituyen un factor que innegablemente complican la conformación y reconocimiento de este vínculo, precisamente por la dificultad de establecer todas las dinámicas que la relación presencial posibilita. No es una imposibilidad en sí, pues corresponde al docente buscar las formas en las que éste se pueda generar.

\section{Sexto principio. Atender la tensión: satisfacción-esfuerzo en el proceso de aprender}

Satisfacción y esfuerzo, un reto didáctico que es necesario reconocer, se deriva de la necesidad de que el aprendizaje se mueva en la confrontación de ser un espacio personal de satisfacción, e incluso de disfrute, frente a

\footnotetext{
${ }^{8}$ Consideramos en el vínculo la existencia de elementos internos y externos en los sujetos que se encuentran en el contexto de la enseñanza intencionada. Unos elementos provienen de sus saberes previos, de la información que poseen, así como de las disposiciones que tienen para la situación de aprendizaje que se presenta, mientras que los externos provienen de las condiciones objetivas en las que se genera la posibilidad de aprender.
} 
la exigencia al mismo tiempo de esfuerzo, dedicación y, porque no afirmarlo, la necesidad de desplazar un deseo satisfactorio inmediato y legítimo, para dejar espacio al esfuerzo que requiere desarrollar un aprendizaje.

Iniciemos por el primer término de este par de polos opuestos que confluyen en toda situación de aprender. Construir un aprendizaje requiere vivir una experiencia satisfactoria por parte de los alumnos. El disfrute en el acto de aprender ciertamente obedece a una serie de condiciones y decisiones personales, sin las cuáles éste no se puede dar. Si bien la escuela es una institución social, en una etapa obligatoria para todos los sujetos, también la escuela es el espacio fundamental para la socialización de los individuos. Es la escuela la que inicialmente permite al ser humano tomar contacto con otras formas de convivencia, asumir ciertas normas sociales. Sin embargo, en el caso específico de la educación superior, existe de por medio una decisión personal de quien busca estar en ella. No se es alumno universitario por obligación, lo que no significa que no tenga obligaciones. Hay una decisión personal subyacente al estar cursando estudios profesionales. Se ha elegido prepararse para una profesión con base en muchos elementos que se han tomado en cuenta para esta decisión, entre ellos, se encuentra el gusto para lograr una formación profesional e incluso el gusto por alguna de las disciplinas que forman parte de ella.

$\mathrm{El}$ aprendizaje que se realiza en cualquier etapa de la formación reclama cierto monto de satisfacción, tema que es intrínseco en el caso de la educación superior. Corresponde a un docente pensar en la necesidad de que el alumno experimente el goce de aprender. No se trata, como se suele postular en los proyectos de uso de tecnologías digitales, de partir de crear jueguitos que entretengan al estudiante; varios proyectos de lo que actualmente se denomina "gamificación de la educación" responden a esta idea simple. Se trata, por el contrario, de algo mucho más complejo, de crear un ambiente de aprendizaje que al mismo tiempo que representa un reto y una exigencia, permita que el alumno encuentre satisfacción e incluso gozo en aprender. No se puede confundir esta perspectiva con una visión infantilizante del trabajo escolar, sino que ésta surge de la necesidad de dar a conocer los sentidos que pueden tener los proyectos de trabajo, de trabajar para que los alumnos construyan el aprendizaje como un proyecto personal (Meirieu, 2002), y que una vez realizado el esfuerzo que reclama el desarrollo de este proyecto, el estudiante encuentre que junto con esta dedicación logra una satisfacción personal y también grupal.

$\mathrm{El}$ docente puede ayudar a estimular este proceso en la forma de promover el trabajo de ciertos contenidos del curso escolar, con las estrategias que impulsa para su desarrollo, así como en su disposición para pensar en las condiciones de sus estudiantes y, sobre todo, para comprender la reacción de ellos ante cada situación didáctica específica. Reconocemos que lograr esta situación no es fácil en el trabajo mediado por tecnologías digitales.

Junto a la necesidad de fomentar el disfrute en el acto de aprender, también es necesario reconocer que aprender requiere de esfuerzo, de dedicación, de vencer dificultades. El aprendizaje no es un acto mecánico que se vincula automáticamente con ninguna acción de enseñanza. Al respecto, Basabe et al. (2007) plantean que no hay una relación directa entre la acción de transmisión y el aprendizaje que realizan los estudiantes. Que éstos pueden no realizar un aprendizaje o el aprendizaje pretendido, o incluso pueden realizar un aprendizaje que sea diverso al buscado.

Entonces, aprender es el resultado de un esfuerzo individual y en ciertas ocasiones grupal, ${ }^{9}$ no es un

\footnotetext{
${ }_{9}^{9}$ Desde el desarrollo del pensamiento educativo, no necesariamente pedagógico, asociado a las tecnologías digitales, se suele hablar de trabajo colaborativo, enfatizando el término colaboración entre diversos actores de aprendizaje. En lo personal prefiero emplear el término grupal, primero porque hace referencia a la forma como se estructura el grupo escolar y las posibilidades de reorganizarlo en trabajo en pequeños grupos; segundo, porque responde mejor a una tradición didáctica, que al menos se puede rastrear desde la Ratio
} 
acto pasivo de un sujeto o individuos, que sin hacer nada logran aprender algo, sino responde a la intencionalidad que cada alumno tenga por trabajar para construir ese aprender, de ahí la necesidad de que el estudiante construya su proyecto de aprendizaje. Un proyecto personal, que vale la pena realizar, pero que para concretar su logro requiere no sólo reconocer una meta, sino al mismo tiempo requiere dedicar tiempo y asumir los esfuerzos para su consecución. Reconocer la necesidad de que el estudiante realice el acto de aprender a partir de un esfuerzo, no significa que el docente sea insensible al mismo, que sólo asuma el papel de exigir el trabajo de sus estudiantes, sino que su tarea requiere, por una parte, comprender las dificultades o retos que se van presentando en cada etapa del trabajo que realizan sus alumnos y, por otra parte, alentarlos a seguir adelante.

Combinar en el trabajo docente exigencia, impulso hacia la construcción de un proyecto de aprendizaje y al mismo tiempo que ese acto sea significativo y satisfactorio para sus estudiantes no es tarea sencilla. Significa partir de principios didácticos que no son fácilmente asimilables. El postulado didáctico formulado desde Comenio sigue vigente: la voluntad del alumno no puede ser violentada.

El señalamiento de estos principios didácticos puede considerarse como la base para iniciar la necesaria re-invención de la docencia en la educación superior. Su desglosamiento no agota el debate didáctico, ni tampoco regresa el planteamiento que emerge de esta disciplina a una serie de normas. $\mathrm{Al}$ contrario, estos principios abren al docente opciones que pueden orientar su trabajo, pero corresponde a cada docente, frente a su compromiso profesional y su indispensable autonomía, decidir la forma como los interpreta y lleva al aula.

Este planteamiento muestra que pensar la docencia de la pospandemia sólo como la inclusión de tecnologías digitales es insuficiente. Este planteamiento didáctico de ninguna manera cancela la necesidad de pensar los problemas estructurales que enfrenta la universidad con los que hemos iniciado este ensayo.

\section{A manera de conclusión}

La universidad es una institución social que siempre se ha adecuado a las condiciones de su época, igualmente la docencia ha sufrido cambios importantes en los diferentes periodos históricos. En este sentido, resulta preocupante que una vez que la pandemia provocó la suspensión de diversas actividades universitarias, en particular su función de trabajo presencial, la universidad no utilice este espacio para repensar su finalidad, su estructura, su forma de vincularse con la sociedad, en el contexto actual. Que no reconozca las diversas dimensiones de su crisis.

La pandemia permitió mostrar los rostros de diversas crisis institucionales que ya estaban presentes previamente, pero lejos de emplear este tiempo la universidad y los universitarios para analizar la forma como sus fines han quedado supeditados a la obtención de puntos para mostrar evidencias de "su calidad", la respuesta dada muestra no sólo conformidad con ello, sino una tensión entre limitarse a una mera modernización o buscar un cambio estructural como en otras crisis la institución ha buscado llevarlo a cabo. Todo indica que la respuesta se está orientando sólo a su modernización, la inclusión de tecnologías digitales ayuda en ello.

Ciertamente la institución dio una respuesta instrumental necesaria para no cancelar absolutamente su tarea docente, se apoyó como nunca antes lo había hecho en la tecnología digital. Esta respuesta fue la única posible en el contexto de la pandemia, pero al mismo tiempo mostró la desigualdad social y la desatención que se había dado al uso de la tecnología

Studiorum de la Compañía de Jesús (1594), varios planteamientos en el texto de Comenio (1657) (forme decurias), propuestas de la escuela lancasteriana y, en particular, el desarrollo de la estrategia didáctica del trabajo por proyectos del movimiento escuela activa ya en el siglo XX. Por otra parte, por la necesidad de reconocer que en un grupo escolar existe lo que diversos autores de corrientes grupales señalan como un "fenómeno grupal", fenómeno que necesariamente requiere ser objeto de interpretación por parte del docente. 
digital para la educación. En todo momento se tuvo que recurrir a plataformas que devengan un costo para la institución, pues las diversas instituciones universitarias nunca generaron una propia. $\mathrm{Al}$ mismo tiempo mostró una cara de la desigualdad social al dejar fuera un importante número de estudiantes que no tienen las condiciones requeridas para realizar el trabajo digital que se requiere para continuar sus estudios.

Mostramos en particular como en los años setenta del siglo pasado, a partir de la crisis generada por el movimiento del 68, la universidad mexicana generó diversas experiencias de transformación tanto de su organización, como de su trabajo docente. Un intento de hacer una modificación de fondo, cuestión que en este momento está desaprovechando. En el ensayo reconocemos también la necesidad de un cambio profundo en el trabajo docente. Un cambio que implique una transformación de su tarea docente, pero que no se limite al uso de tecnologías digitales sin necesariamente excluirlas.

Así, se ha mostrado como la actividad docente se ha alejado del debate didáctico contemporáneo. La didáctica, como la disciplina pedagógica que analiza los procesos que se dan en la transmisión intencionada del conocimiento, la didáctica como una disciplina que se ha ignorado ampliamente en el trabajo universitario.

En el siglo XXI ya no se habla de la cátedra como elemento organizador de la enseñanza universitaria, pero la clase expositiva sigue siendo la técnica

\section{Referencias}

Aebli, H. (1958), Una didáctica fundada en la psicología de Jean Piaget, Buenos Aires, Kapalusz.

Agamben, G. (2020), "Requien por los estudiantes", Roma, Instituto Italiano Per gli Studi Filosofici, $<$ https:/ / artilleriainmanente.noblogs.org/?p=1514> [Consulta: enero de 2021]. privilegiada para el trabajo en el aula, muchas de las prácticas que se promueven en la llamada educación a distancia, reproducen de otra manera esta relación. Se postula en ellas lecturas presentadas en PDF, videos de YouTube seguidos de una serie de preguntas que el alumno debe resolver. La clase expositiva o las lecturas en el trabajo a distancia son el instrumento primordial para que los alumnos se alleguen a la información.

La renovación didáctica es urgente. Pero de una didáctica que lejos de establecer normas y pautas de trabajo para los docentes, parta de principios que ayuden al docente a reflexionar sobre cómo construye un ambiente de aprendizaje. De suerte que al ayudarles a pensar de qué otras formas pueden organizar la transmisión intencionada de contenidos para el aprendizaje, la pospandemia se constituya en un punto de inflexión. Esto significa reivindicar la responsabilidad del docente en la organización del trabajo en el aula.

Este ensayo invita a re-pensar la universidad y al mismo tiempo re-fundar la tarea docente a partir, en este último caso, de un conjunto de principios didácticos, los que en el marco de la autonomía indispensable para el trabajo docente permitan orientar un cambio fundamentado en un pensamiento didáctico que conjugue los saberes profesionales, curriculares y experienciales. Re-inventar el trabajo docente en donde las tecnologías digitales puedan ser un instrumento pero no el fundamento de esa transformación. 
Coll, G (2013) "La educación formal en la nueva ecología del aprendizaje, tendencias, retos y agenda de investigación", en J. Rodríguez (ed.), Aprendizaje y educación en la sociedad digital, Barcelona, Universidad de Barcelona, en [https://www.researchgate.net/ publication/259006556_La_educacion_formal_en_ la_nueva_ecologia_del_aprendizaje_tendencias_ retos_y_agenda_de_investigacion].

Comenio, J. A. (1982), Didáctica Magna, México, Porrúa (Sepán Guantos).

Chevallard, Y. (1997), La transposición didáctica. Del saber sabio al saber enseñando, Buenos Aires, Aique.

Díaz Barriga, A. (2020) "De la integración curricular a las políticas de innovación en la educación superior mexicana", Perfiles Educativos, vol. 42, núm. 169, pp. 160-179 [http://perfileseducativos.unam.mx/iisue_ pe/index.php/perfiles/article/view/59478/52528].

Díaz Barriga, A. (en prensa), "Reinventar la docencia, una tarea para el siglo XXI. La didáctica ante la sobreestimulación de los sujetos de la educación", en A. Díaz-Barriga (coord.), Docente y didáctica. Acercamientos polémicos, México, IISUE-UNAM.

Dilthey, W. (1965), Fundamentos de un sistema de pedagogía, Buenos Aires, Losada.

Durkheim, E. (1992), Historia de la educación y de las doctrinas pedagógicas, Madrid, La Piqueta.

Foucault, M. (2009), Vigilar y castigar. Nacimiento de la prisión, México, Siglo XXI.

Freinet, C. (2009), Consejos a los maestros jóvenes, México, Fontamara.

Freinet, C. (1993), Técnicas Freinet para la escuela moderna,
México, Siglo XXI.

Freitag, M. (2004), El naufragio de la universidad, Barcelona, Pomares-Corredor.

González Casanova, P. (1976), "Carta al consejo editorial”, Cuadernos Políticos, núm. 10, octubre-diciembre, pp. 94-112 [http://www.cuadernospoliticos.unam.mx/ cuadernos/contenido/CP.10/CP10.12.Cartaeditorial. pdf].

Keller, F. (1968), “Goodbye, teacher...”, fournal of Apllied Behavior Analysis, núm. 1, Spring, pp. 79-89, en <https:// www.ncbi.nlm.nih.gov/pmc/articles/PMC1310979/ pdf/jaba00083-0078.pdf>.

Instituto de la Educación Superior para América Latina y el Caribe (IESACL) (2020), COVID-19 y educación superior. De los efectos inmediatos al día después, [http://grupomontevideo.org/sitio/wp-content/ uploads / 2020/04/Informe-IESALC-UNESCOCOVID19.pdf].

Meirieu, P. (2002), Aprender sí. Pero ¿cómo?, Barcelona, Octaedro.

Meirieu, P. (2006), Carta a un joven profesor, Barcelona, Grao. Pichón-Rivière, E. (1980), La teoría del vínculo, Buenos Aires, Nueva Visión.

Sánchez, S. (2007), "Escritura y revolución. Una historia política de Los Enfermos a través de sus producciones discursivas", Revista Letras Históricas, núm. 4, enerojunio 2011, pp. 161-180 [http://publicaciones.cucsh. udg.mx/pperiod/Lhistoricas/pdfs/vol4/7.pdf].

Snyders, G. (1972), Pedagogía progresista, Madrid, Marova.

Tardif, M. (2010), Los saberes del docente y su desarrollo profesional, Madrid, Narcea.

Cómo citar este artículo:

Díaz-Barriga, Ángel (2021), "Repensar la universidad: ir más allá de la inclusión de tecnologías digitales", Revista Iberoamericana de Educación Superior (RIES), vol. XII, núm. 33, pp. 3-20, DoI: https://doi.org/10.22201/iisue.20072872e.2021.34.976 [Consulta: fecha de última consulta]. 